02

\title{
Особенности оптических и энергетических свойств тонких пленок CdSe
}

\author{
() Г.А. Ильчук ${ }^{1}$, Р.Ю. Петрусь ${ }^{1}$, А.И. Кашуба ${ }^{1,2}$, И.В. Семкив ${ }^{1}$, Э.О. Змийовська ${ }^{1}$ \\ ${ }^{1}$ Национальный университет „Львовская политехника“, \\ 79013 Львов, Украина \\ 2 Львовский национальный университет им. Ивана Франко, \\ 79005 Львов, Украина \\ e-mail: ilchukg@gmail.com
}

Поступила в редакцию 13.06.2019 г.

В окончательной редакции 13.06.2019 г.

Принята к публикации 23.09.2019 г.

Представлены результаты экспериментальных и теоретических исследований оптических и энергетических свойств тонких пленок CdSe, полученных методом квазизамкнутого объема. Приведены методика синтеза, результаты структурных и оптических исследований тонких пленок $\mathrm{CdSe}$, осажденных на поверхности кварцевой подложки марки КВ (ГОСТ 15130-86). Качество пленок анализируется с использованием методики рентгеновской дифракции, энергодисперсионного анализа и сканирующей электронной микроскопии. В рамках метода псевдопотенциала теоретически изучена динамика изменения параметров электронной подсистемы пленки CdSe. Установлен прямозонный характер запрещенной щели пленки. На основе плотности состояний установлен генезис зоны проводимости и валентной зоны. С использованием соотношения Крамерса-Кронига получены спектры оптических диэлектрических функций, отражения, показателя преломления и экстинкции, которые удовлетворительно коррелируют с полученными экспериментальными данными.

Ключевые слова: тонкая пленка, электронный энергетический спектр, плотность состояний, оптические функции, запрещенная зона, интерференция.

DOI: $10.21883 /$ OS.2020.01.48837.215-19

\section{Введение}

Полупроводниковые пленки $\mathrm{CdSe}$ являются важным материалом группы $\mathrm{A}^{\mathrm{II}} \mathrm{B}^{\mathrm{VI}}$ для использования в современных электронных устройствах, таких как солнечные элементы, светодиоды, газовые сенсоры и прочее $[1,2]$. Синтез тонких пленок CdSe, получение и понимание физической информации об их структурных, оптических и электронных свойствах является необходимым условием разработки стабильных устройств на их основе.

Для получения пленок селенида кадмия используют различные методы (высокочастотное магнетронное распыление [3], газотранспортный метод (PVT) [4], химическое парофазное осаждения (CVD) [5], молекулярнолучевая эпитаксия [6], термическое вакуумное испарение [7] и термическое испарение в квазизамкнутом объеме (КЗО) [8]).

Получение бинарных полупроводниковых пленок $\mathrm{A}^{\mathrm{II}} \mathrm{B}^{\mathrm{VI}}$, в частности $\mathrm{CdSe}$, в условиях вакуума осложняется различием давлений насыщенного пара компонентов соединения. Также важным параметром синтеза пленок в вакууме является уровень остаточных газов, которые способны вступать в химические реакции с веществом подложки и входить в решетку кристаллитов. Они, как правило, неконтролируемо влияют на скорость роста пленок, их структуру, электрофизические и оптические свойства. Поэтому выращивание полупроводни- ковых пленок из паровой фазы необходимо проводить в дегазированной вакуумной системе с остаточным давлением химически активных газов не более $10^{-5} \mathrm{~Pa}$.

Выбор метода КЗО в этой работе обусловлен тем, что он позволяет получать однородные, структурно совершенные тонкие пленки полупроводниковых материалов и их твердых растворов с близким по своему составу к стехиометрическому в условиях, близких к термодинамическому равновесию, что является основным преимуществом данного метода перед другими вакуумными технологиями $[9,10]$. Важным преимуществом метода является высокое давление паров халькогенидов в КЗО, значительно превышающее (на четыре порядка) давление остаточных газов в рабочем объеме [11]. Это предотвращает уменьшение неконтролируемого легирования выращенных пленок остаточной атмосферой рабочей камеры [12-14]

В научных публикациях последних лет большое внимание уделяется определению оптимальных условий роста тонких пленок CdSe [15] и исследованию электрических и оптических свойств с целью улучшения характеристик устройств на их основе [16], в частности, тонкопленочных солнечных элементов на основе фотоактивного слоя CdTe [17]. Последние работы сосредоточены на использовании слоя CdSe в качестве партнера $\mathrm{CdTe}$. Использование слоя $\mathrm{CdSe}\left(E_{g}=1.7 \mathrm{eV}\right)$ приводит к его диффузии в $\mathrm{CdTe}$, превращая приповерхностную 
область в твердый раствор замещения $\mathrm{CdTe}_{1-x} \mathrm{Se}_{x}$. Это приводит к расширению спектрального рабочего диапазона и, как следствие, увеличению тока короткого замыкания солнечного элемента [17].

Исследование электронного энергетического спектра проводилось достаточно длительный срок [18-21], однако только для монокристаллических образцов. В этих работах указывается на высокое сходство экспериментальных и теоретических данных. Поэтому была поставлена задача расчета электронного энергетического спектра тонкой пленки $\mathrm{CdSe}$, а также оптических функций на основе полученного спектра.

Для создания гетероструктур на основе пленок с заданными физическими свойствами важно иметь сведения о кристаллической, энергетической структуре изучаемого материала. Поэтому в работе представлено сравнение теоретических расчетов и экспериментальных исследований структурных и оптико-энергетических свойств тонких пленок $\mathrm{CdSe}$, синтезированных методом КЗО.

\section{Экспериментальная часть}

\section{1. Осаждение тонких пленок CdSe методом квазизамкнутого объема}

Синтез пленок CdSe проводили на кварцевых очищенных подложках марки КВ (ГОСТ 15130-86) толщиной $1 \mathrm{~mm}$ методом термического испарения кристаллического порошка CdSe полупроводниковой чистоты в квазизамкнутом объеме в условиях остаточного давления газа $P=2 \cdot 10^{-5} \mathrm{~Pa}$.

В процессе синтеза области источника и осаждения находились в изотермических условиях, что позволило обеспечить равномерный переход материала в паровую фазу и его осаждение в зоне кристаллизации в условиях, близких к термодинамическому равновесию, что обеспечивает необходимые кристаллографические и электрофизические свойства пленок.

Установка осаждения полупроводниковых пленок методом КЗО (рис. 1) состоит из вакуумной системы безмасляной откачки на основе магнитодиодного насоса фирмы „Varian“ (Италия), в которую герметично подключается кварцевый реактор, что формирует квазизамкнутый объем, и двухзонной печи резистивного нагрева с программным управлением температурными режимами. Кварцевый реактор размещали в вертикальной двухзонной печи (3). Верхняя зона печи контролировала температуру подложки (2), а нижняя - температуру источника (4). В установке предусмотрена возможность включения еще двух дополнительных управляемых нагревателей для работы с несколькими источниками распыления. Кварцевый реактор перед загрузкой исходными материалами химически травили в растворе $1: 1: 1$ фтористоводородной и азотной кислот ОСЧ в бидистиллированной воде в течение $10 \mathrm{~min}$ с последующим многократным промыванием бидистиллированной

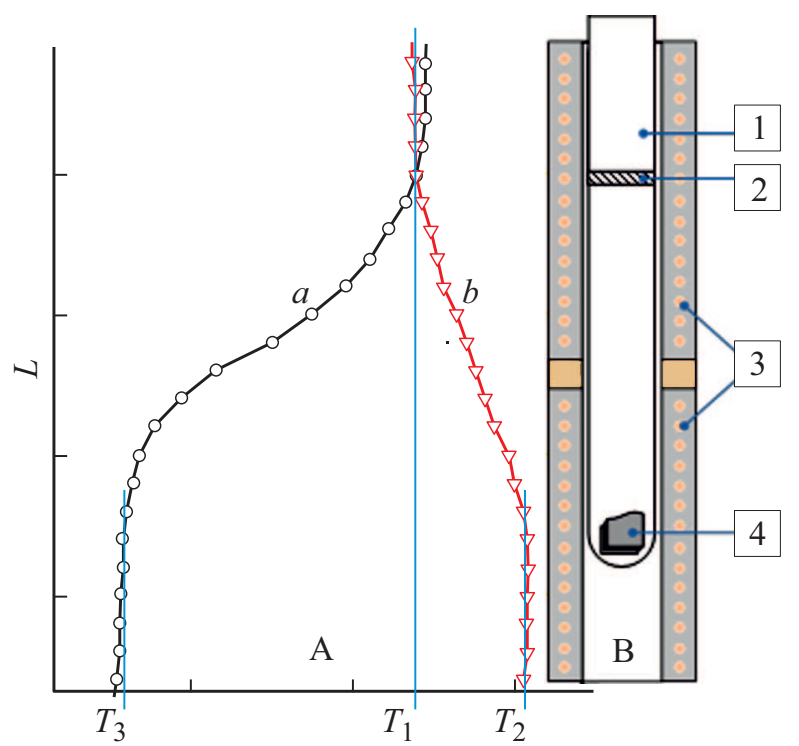

Рис. 1. Принципиальная схема распределения температур А ( $a$ - режим предварительного прогрева подложки, $b-$ режим ростовых условий) и схема вертикальной двузонной печи с реактором КЗО В для роста полупроводниковых пленок.

водой. В дальнейшем ампулу сушили, а на конечном этапе прожаривали в вакууме при температуре $1200 \mathrm{~K}$.

Процесс выращивания пленок на первом этапе предусматривал экспозицию зоны подложки в течение $15 \mathrm{~min}$ при температурах, превышающих температуру зоны источника, с целью предростовой очистки поверхности подложки. Второй этап - рост пленки длился $10 \mathrm{~min}$. На этом этапе подложка находилась при более низких температурах, чем температура источника.

Для получения пленок CdSe устанавливали температурные режимы $T_{1}=700 \mathrm{~K}, T_{2}=900 \mathrm{~K}$. Температурные режимы роста $\left(T_{1}, T_{2}\right)$ избраны на основе термодинамического анализа состава паровой фазы и процессов массопереноса, а также предусматривали проведение процесса выращивания в условиях, близких к равновесным.

\section{2. Методы структурных исследований}

Массивы экспериментальных интенсивностей и углов отражения от исследуемых образцов получены на дифрактометре STOE STADI P при комнатной температуре. Обработку экспериментальных дифракционных массивов, расчет теоретических дифрактограмм, рентгеновский фазовый анализ, индексирование параметров элементарных ячеек проводили с помощью пакета программ STOE WinXPOW и программы PowderCell [22].

Морфологию поверхности и элементный состав пленок CdSe исследовали с использованием растрового электронного микроскопа РЭМ-106И („Selmi“‘, Сумы) с энергодисперсионным рентгеновским анализатором. Проведенные исследования подтвердили формирование соединения CdSe. 

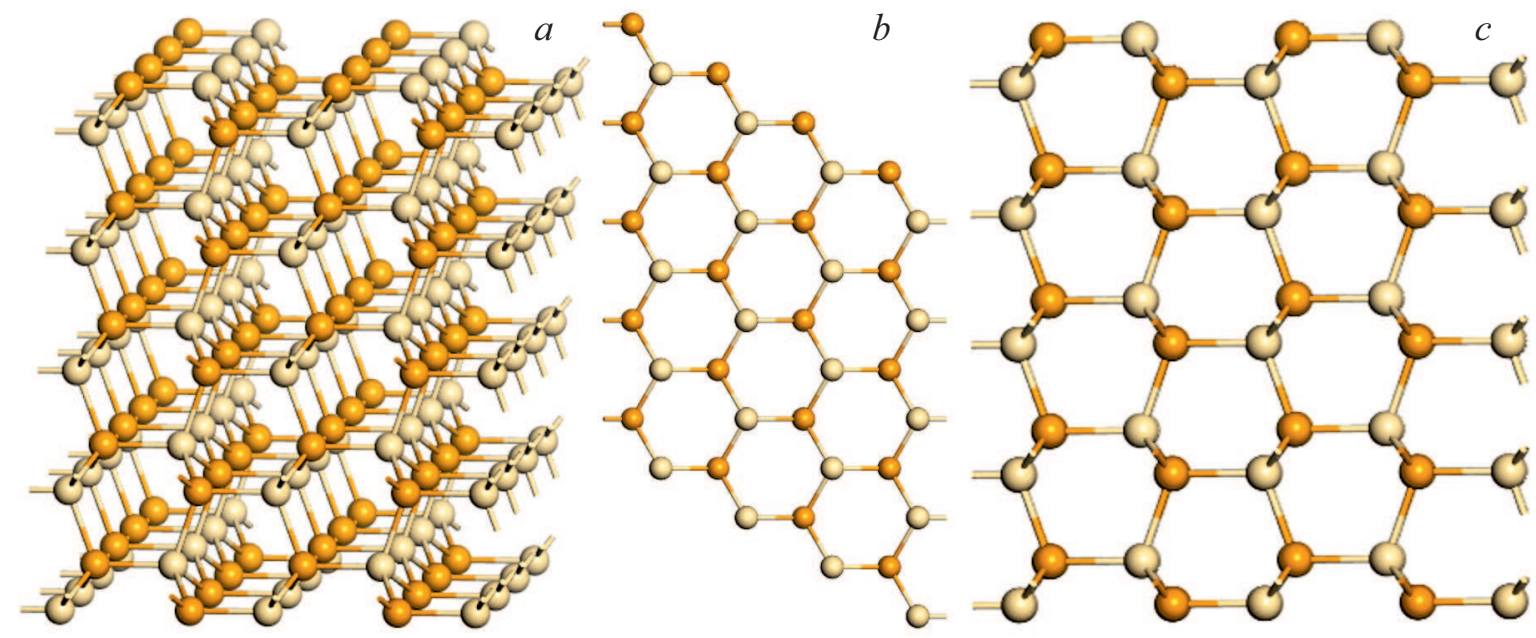

Рис. 2. Кристаллическая структура тонкой пленки CdSe при комнатной температуре в различных плоскостях (Cd - светлые, $\mathrm{Se}-$ темные атомы): $a-(1,1,1) ; b-(1,1,0) ; c-(0,1,1)$.

\section{3. Методика оптических исследований и определения толщины пленки CdSe}

Исследование спектральной зависимости оптического пропускания в видимой и ближней инфракрасной области проведено с использованием спектрофотометра Shimadzu UV-3600. Все оптические исследования проводились при комнатной температуре.

Для определения толщины исследуемых пленок можно использовать уравнение:

$$
d=\frac{M \lambda_{1} \lambda_{2}}{2\left(n\left(\lambda_{1}\right) \lambda_{2}-n\left(\lambda_{2}\right) \lambda_{1}\right)},
$$

где $\lambda_{1}$ и $\lambda_{2}$ - длины волн, соответствующих соседним экстремальным точкам на спектре пропускания, $M=1$ для двух соседних экстремумов одного типа ( $\max -\max$, $\min -\min )$ и $M=1 / 2$ для двух соседних экстремумов противоположного типа $(\max -\min , \min -\max )[23,24]$. Значение показателя преломления взято из его спектральной зависимости, приведенной в [25]. Среднее значение толщины пленки $\mathrm{CdSe}$, рассчитанное по уравнению (1), для всех комбинаций экстремальных точек составляет $1.875 \mu \mathrm{m}$. Погрешность определения толщины установлена с использованием среднеквадратического отклонения и составляет $11.7 \%$.

Толщину пленок измеряли также на профилометpe Veeco, модель Dektak 8. Установлено, что средняя скорость осаждения пленок составляла $3.1 \mathrm{~nm} / \mathrm{s}$, а полученные значения толщины находятся в пределах приведенной выше погрешности.

\section{4. Методика расчета}

Теоретические расчеты проведены в рамках теории функционала электронной плотности (DFT). Для расчета энергетических и оптических свойств тонких пленок
$\mathrm{CdSe}$ использованы структурные параметры кристаллической решетки монокристаллического образца [18]. Модели пленки $\mathrm{CdSe}$ формировали из объемного кристалла $\mathrm{CdSe}$ вдоль оси [001]. Расстояние между формульными единицами увеличивалось приблизительно на $15 \mathrm{~nm}$. Таким образом, формируется вакуумная щель, чтобы избежать взаимодействия между слоями. Толщина тонкой пленки составляла приблизительно $9.5 \mathrm{~nm}$, что меньше чем в экспериментально синтезированных пленках $\mathrm{CdSe}$. Поскольку $a b$ initio расчеты пленок толщиной порядка нескольких микрометров требуют значительных компьютерных ресурсов, представленные ниже результаты могут отражать влияние размерного эффекта на свойства пленки.

Для ионных потенциалов использовались ультрамягкие псевдопотенциалы Вандербильта [26]. Для описания обменно-корреляционной энергии электронной подсистемы использовался функционал в приближении обобщенного градиента (GGA) в параметризации Пердью, Бурке и Ернцергофа (PBE) [27]. Для корреляционного потенциала к границам высокой плотности применены формула Кеперли-Алдера и соотношение Гелл-МанаБракнера.

Для расчетов использовали значение энергии обрезания плоских волн $E_{\text {cut-off }}=260 \mathrm{eV}$. Сходимость общей энергии составляла около $1 \cdot 10^{-6} \mathrm{eV}$. Электронная конфигурация для атомов такова: $\mathrm{Cd}-\mathrm{Kr} 4 d^{10} 5 s^{2}$, $\mathrm{Se}-\mathrm{Ar} 4 s^{2} 4 p^{4}$, где состояния $[\mathrm{Kr}]$ и $[\mathrm{Ar}]$ принадлежат к остову. Интегрирование по зоне Бриллюэна выполнялось по сетке $4 \times 1 \times 2 k$-точек с помощью схемы Монхорста-Пака [28].

Оптимизацию атомных координат и параметров элементарной ячейки проводили с использованием метода Бройдена-Флетчера-Гольдфарба-Шани (BFGS) [29]. Оптимизацию продолжали до тех пор, пока силы, действующие на атомы, не стали меньше $0.01 \mathrm{eV} / \AA \AA$, максимальное смещение $10^{-5} \AA$, а механические напряжения в ячей- 
$a$

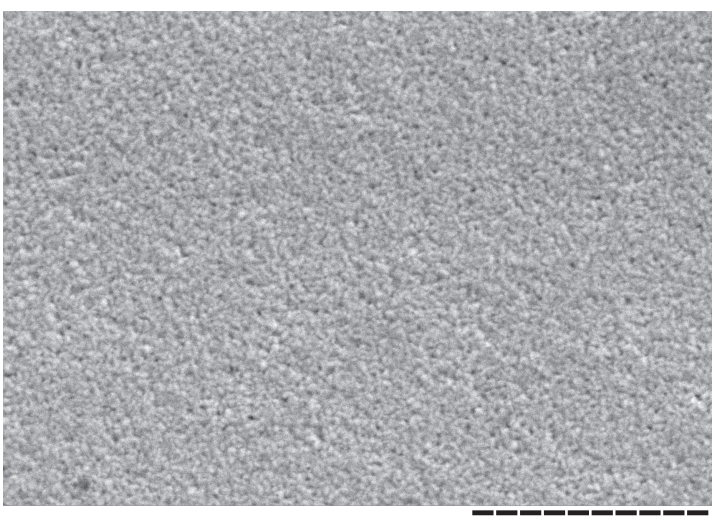

$\mathrm{WD}=24.9 \mathrm{~mm} 20.00 \mathrm{kV} \times 2.00 \mathrm{k} 20 \mu \mathrm{m}$

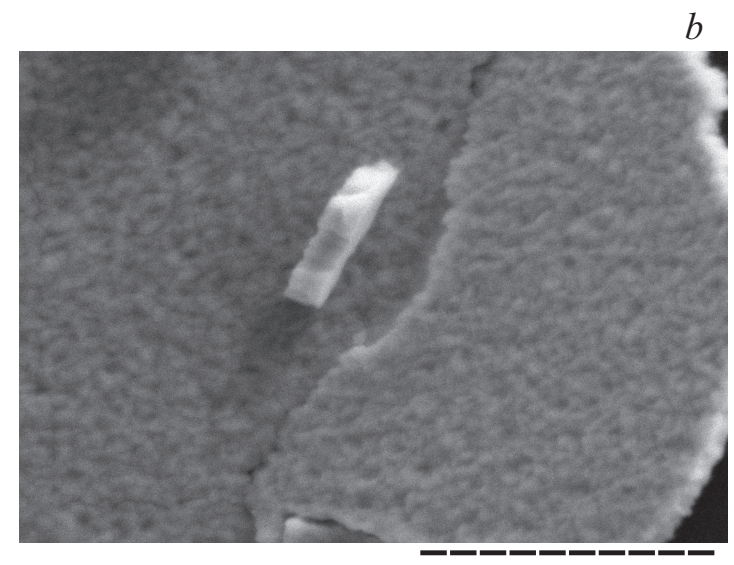

$\mathrm{WD}=24.9 \mathrm{~mm} 20.00 \mathrm{kV} \times 5.00 \mathrm{k} \quad 10 \mu \mathrm{m}$

Pис. 3. Морфология поверхности пленки CdSe.

ке - меньше $0.02 \mathrm{GPa}$. Зонно-энергетическую диаграмму построили по точкам зоны Бриллюэна в обратном пространстве Г $(0,0,0) ; \mathbf{F}(0,0.5,0) ; \mathbf{Q}(0,0.5,0.5)$; $\mathbf{Z}(0,0,0.5)$.

\section{Результаты и обсуждение}

B процессе синтеза получены пленки $\mathrm{CdSe}$ серой окраски. Морфология поверхности полученных пленок CdSe приведена на рис. 3. Установлено, что подложка равномерно покрыта кристаллитами соединения $\mathrm{CdSe}$ (рис. 3,a). Поверхность характеризуется однородным распределением зерен по размерам, это позволяет сделать вывод, что рост по всей плоскости подложки был в однородных условиях. Проведя тонкий скол пленки, с помощью растрового электронного микроскопа определена толщина пленки, которая составляет около $1.3 \mu \mathrm{m}$ (рис. 3,b). Данное значение хорошо согласуется с результатами оптических и механических исследований (отклонение находится в пределах погрешности).

Из анализа дифрактограммы для CdSe установлено, что пленка однофазная. Пространственная группа соединения $\mathrm{CdSe}-P 6_{3} m c$, что является характерным для селенида кадмия [18].

На рис. 4 приведен спектр оптического пропускания $T(\lambda)$, полученный при комнатной температуре для пленок CdSe. Виден четко выраженный край фундаментального поглощения, который локализован в окрестности $740 \mathrm{~nm}$, что является подтверждением наличия соединения селенида кадмия. Также пленка характеризуется высоким значением пропускания (более 50\%).

Спектры пропускания характеризуются наличием экстремумов интерференции, что свидетельствует о совершенстве полученных пленок. Наличие интерференционных максимумов и минимумов в диапазоне длин волн от 750 до $2500 \mathrm{~nm}$, определяется набегом фазы на $2 \pi$ при изменении длины световой волны.

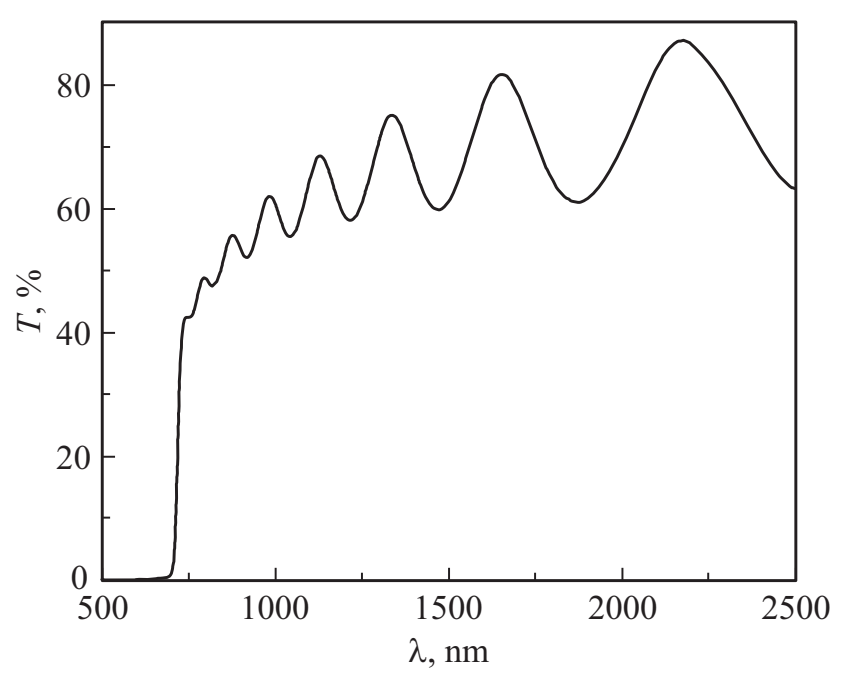

Рис. 4. Спектр пропускания пленки CdSe.

Спектральная зависимость поглощения пленок $\mathrm{CdSe}$ в координатах $(\alpha h v)^{2}-h v$ демонстрирует наличие края фундаментального поглощения (рис. 5). Ширина запрещенной зоны пленок CdSe определялась путем экстраполяции линейной части спектральной зависимости квадрата коэффициента поглощения $(\alpha h v)^{2}-f(h v)$ к оси энергии фотонов [14,30]. Определенное значение оптической ширины запрещенной зоны пленки CdSe составляет $1.68 \mathrm{eV}$. Полученная величина запрещенной зоны хорошо согласуется с литературными значениями [31]. Линейный характер зависимостей $(\alpha h v)^{2}-f(h v)$ в интервале $1.65-1.75 \mathrm{eV}$ указывает на формирование края поглощения прямыми межзонными оптическими переходами.

Ранее [18-21] сообщалось о расчетах электронного энергетического спектра монокристаллического образца селенида кадмия. Для того чтобы иметь возможность сопоставить данные расчета и эксперимента, проведены расчеты для тонкой пленки CdSe. На рис. 6 изобра- 


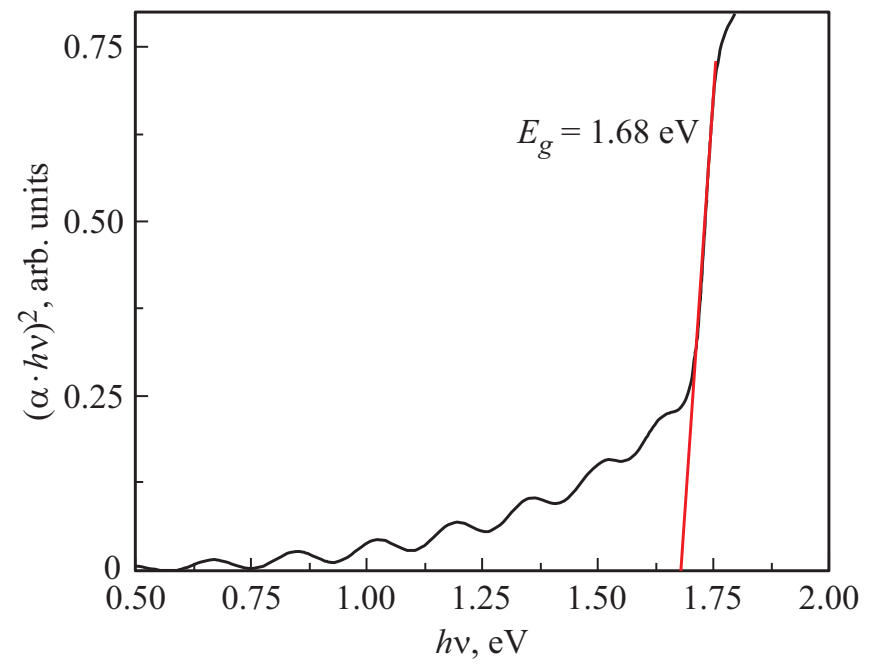

Рис. 5. Спектральная зависимость оптического поглощения пленки CdSe.

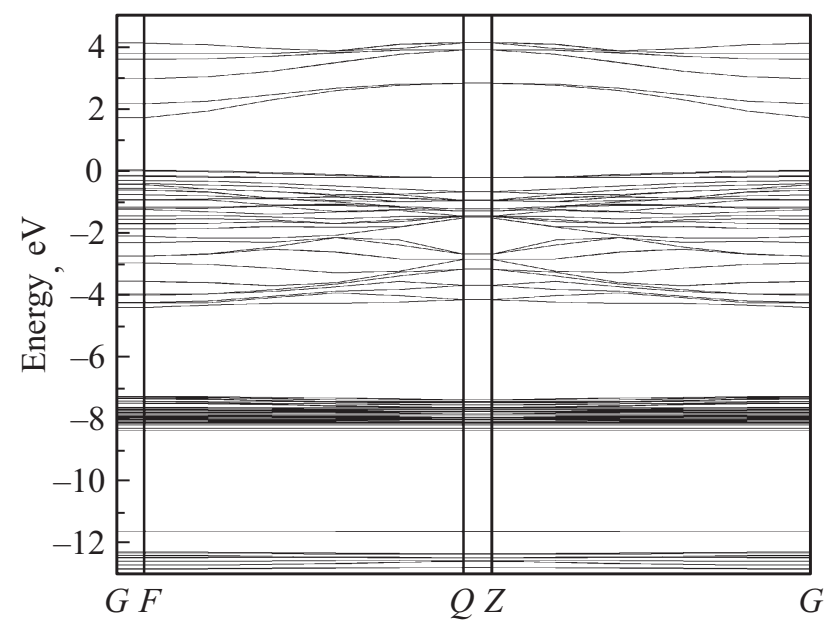

Рис. 6. Зонно-энергетическая диаграмма тонкой пленки CdSe.

жена полная зонная энергетическая диаграмма тонкой пленки CdSe вдоль высокосимметричных линий зоны Бриллюэна. Вершина зоны Бриллюэна, обозначенная как $0 \mathrm{eV}$, совпадает с уровнем Ферми. Запрещенная зона является зоной прямого типа, что предусматривает прямые оптические переходы в точке $\mathbf{G}$ зоны Бриллюэна. Однако вследствие низкой дисперсности энергетических уровней возможны также непрямые переходы с участием фононов, которые не учитываются в расчетах.

Анализ результатов теоретических расчетов зонноэнергетического спектра показывает, что наименьший энергетический промежуток запрещенной зоны локализован в центре зоны Бриллюэна (точка G). Кроме того, можно увидеть четкую разницу анизотропии между валентной зоной и зоной проводимости $E(\mathbf{k})$. Вершина валентного комплекса более плоская, это вызвано тем, что дырки имеют меньшую подвижность в сравнении с электронами [32]. Такое поведение вызвано зависимо- стью эффективной массы электрона/дырки и дисперсии энергетических уровней $E(\mathbf{k})[33]$ :

$$
\frac{1}{m_{*}}=\frac{4 \pi^{2}}{h^{2}} \frac{d^{2} E(k)}{d k^{2}} .
$$

Максимальная дисперсия $E(k)$ наблюдается для полос в направлении $\mathrm{G}-\mathrm{Z}$ и $\mathrm{G}-\mathrm{F}$ зоны Бриллюэна. Анализ парциальных вкладов отдельных уровней в функцию полной плотности состояний (рис. 7) и парциальных вкладов отдельных зон в электронную плотность позволил определить генезис валентных зон и зоны проводимости тонкой пленки CdSe. Самые низкие зоны от -13 до $-11 \mathrm{eV}$ сформированы $s$-состояниями Se. Формирование следующих зон, диспергированных от -9 до $-6 \mathrm{eV}$, происходит от вклада $d$-состояний $\mathrm{Cd}$. Это характерно и для других соединений кристаллов и тонких пленок группы $\mathrm{A}^{\mathrm{II}} \mathrm{B}^{\mathrm{VI}}(\mathrm{A}=\mathrm{Cd}, \mathrm{B}=\mathrm{S}, \mathrm{Te})[14,34]$. Вершина валентного комплекса практически сформирована из $p$-состояний $\mathrm{Se}$ с примесью $p$-состояний $\mathrm{Cd}$. Но дно зоны проводимости в основном сформировано из $p$-состояний $\mathrm{Cd}$ и $\mathrm{Se}$, а также $s$-состояниями $\mathrm{Cd}$.

Учитывая правила отбора, результаты расчетов электронного энергетического спектра и плотности состояний, можем предположить, что наименьшую запрещенную зону формируют прямые $s$-p-переходы. Не исключено, что прямозонный переход (локализованный в точке $\mathbf{G}$ зоны Бриллюэна) может быть сформирован соединением $\mathrm{Cd}-\mathrm{Se}[18]$.

Для исследования оптических свойств удобно использовать комплексную диэлектрическую функцию $\varepsilon(\omega)$. Мнимую часть диэлектрической функции $\varepsilon(\hbar \omega)$ $\left(\varepsilon=\varepsilon_{1}+i \varepsilon_{2}\right)$ в $\mathrm{CdSe}$ (рис. 8) можно рассчитать с использованием следующего соотношения [35]:

$$
\varepsilon_{2}(\hbar \omega)=\frac{2 e^{2} \pi}{V \varepsilon_{0}} \sum_{K, v, c}\left|\left\langle\Psi_{K}^{c}|\hat{\boldsymbol{u}} \cdot \boldsymbol{r}| \Psi_{K}^{v}\right\rangle\right|^{2} \delta\left(E_{K}^{c}-E_{K}^{v}-\hbar \omega\right),
$$

где $V$ - объем элементарной ячейки, $E_{k}-$ энергия полосы, индексы $c$ и $v$ относятся к состояниям

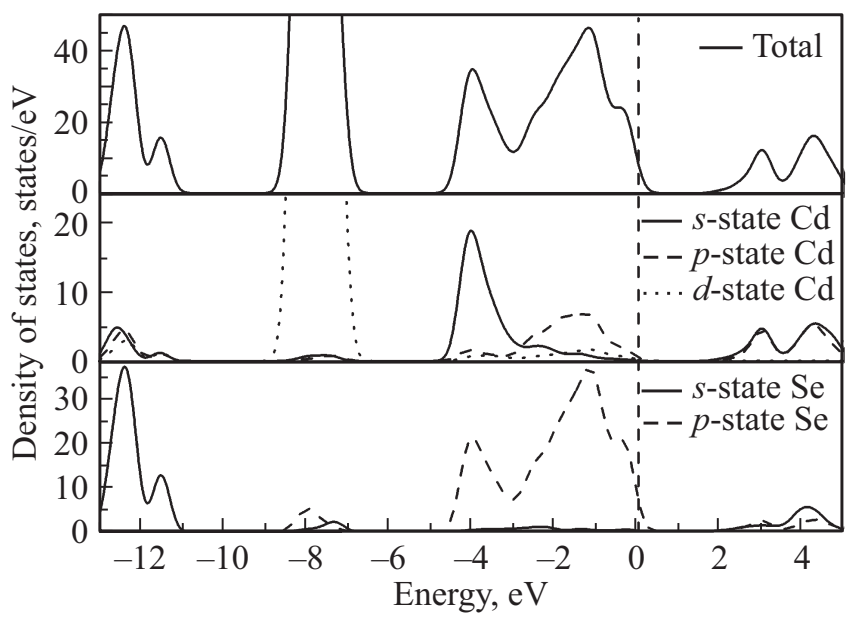

Рис. 7. Плотность состояний тонкой пленки $\mathrm{CdSe}$. 

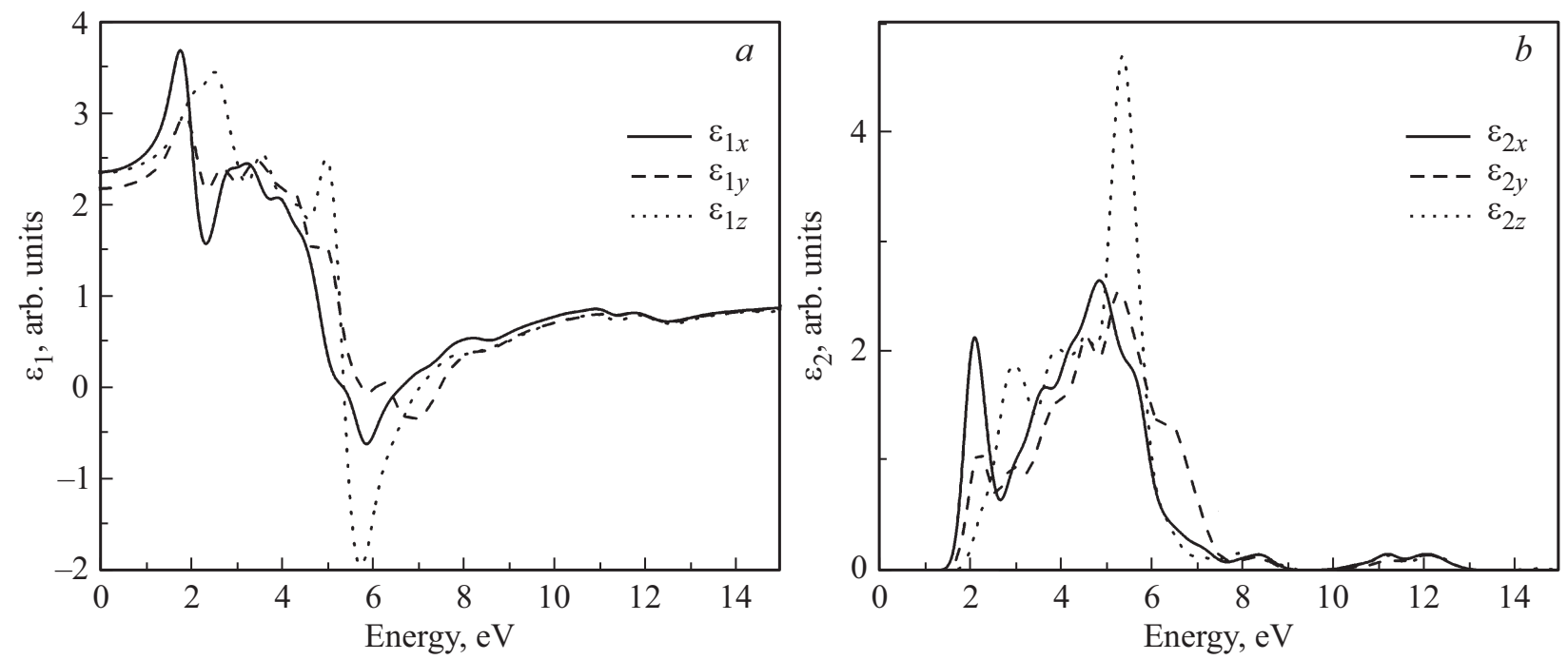

Рис. 8. Действительная $(a)$ и мнимая $(b)$ составляющие функции диэлектрической проницаемости $\varepsilon$ тонкой пленки CdSe в различном кристаллографическом направлении.

проводимости и валентной $k$-зоны соответственно, $u$ - периодическая часть орбитали ячейки (волновых функций) в $k$-точке.

После этого вещественная часть диэлектрической функции $\varepsilon_{1}(\hbar \omega)$ получена с использованием соотношения Крамерса-Кронига:

$$
\varepsilon_{1}(\hbar \omega)-1=\frac{2}{\pi} \int_{0}^{\infty} \frac{t \varepsilon_{2}(t) d t}{t^{2}-(\hbar \omega)^{2}} .
$$

Из кривой мнимой части диэлектрической функции видно, что первая оптическая критическая точка диэлектрической функции, которой является край фундаментального поглощения, возникает при энергии около $1.8 \mathrm{eV}$. При увеличении энергии наблюдается типичный быстрый рост $\varepsilon_{2}(\omega)$ [14,34]. Можем наблюдать значительную анизотропию диэлектрической функции в зависимости от оптической поляризации (рис. 8).

Исходя из расчетов спектра отражения (рис. 9) можем предположить, что пленки $\mathrm{CdSe}$ не чувствительны к ультрафиолетовому излучению как и пленки $\mathrm{CdS}$ [34]. На основе рассчитанных спектров действительной (4) и мнимой частей (3) диэлектрической функции получены спектральные зависимости показателей преломления $(n)$ и коэффициента экстинкции $(k)$ :

$$
\begin{aligned}
& n=\sqrt{\frac{\left(\varepsilon_{1}^{2}+\varepsilon_{2}^{2}\right)^{1 / 2}+\varepsilon_{1}}{2},} \\
& k=\sqrt{\frac{\left(\varepsilon_{1}^{2}+\varepsilon_{2}^{2}\right)^{1 / 2}-\varepsilon_{1}}{2}},
\end{aligned}
$$

Теоретическая дисперсия показателей преломления тонкой пленки $\mathrm{CdSe}$ для видимой области спектра показана на рис. 10, $a$. Видно, что показатели преломления удовлетворяют соотношению $n_{x}>n_{z}>n_{y}$ для

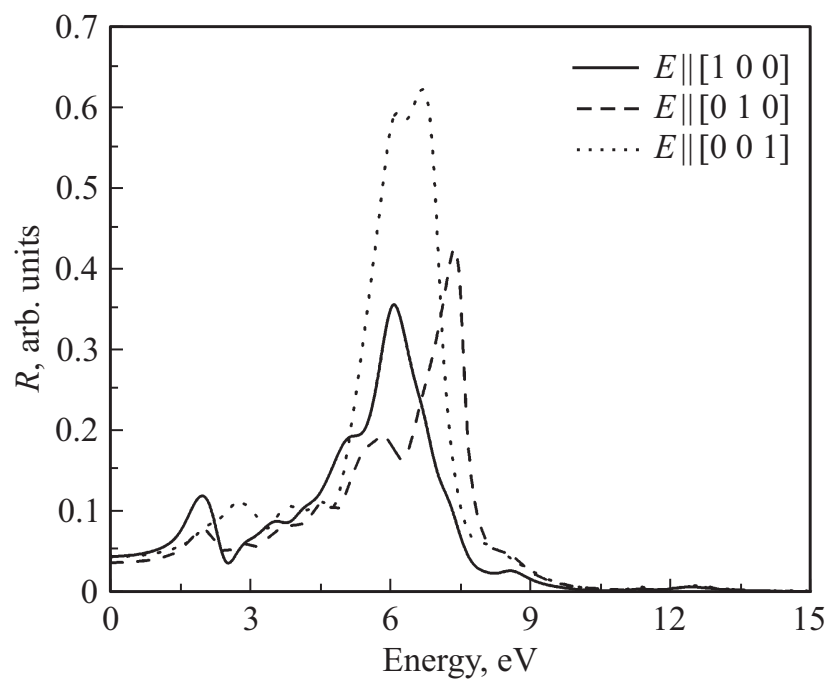

Рис. 9. Теоретически рассчитанные спектры оптического отражения тонкой пленки CdSe.

энергетического диапазона меньше $2 \mathrm{eV}$. В диапазоне между 2 и $6 \mathrm{eV}$ соотношения получает вид $n_{z}>n_{x, y}$ a также есть обратная зависимость в диапазоне $6-10 \mathrm{eV}$. Анизотропия показателя преломления в области выше $10 \mathrm{eV}$ практически не обнаружена. Такое изменение показателя преломления в зависимости от оптической поляризации имеет значение для прикладного использования. Теоретически полученные значения показателей преломления меньше экспериментальных, что может быть вызвано негативным вкладом инфракрасного поглощения в кристалле, которое не учитывалось в расчете (отклонения в пределах 20\%). Анализ коэффициента экстинкции (рис. 10, b) подтверждает наличие анизотропии оптических функций. Это проявляется в смещении 

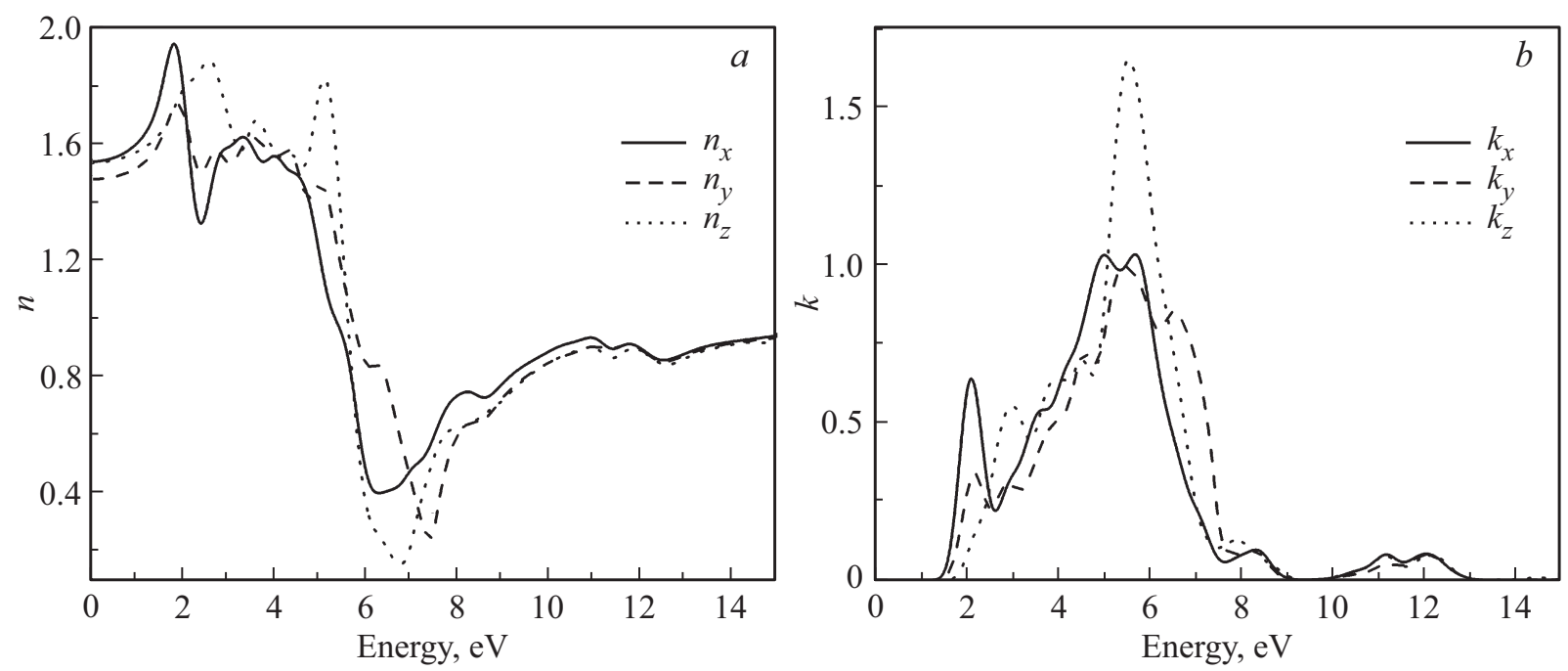

Рис. 10. Теоретически рассчитанные спектры показателей преломления $(a)$ и коэффициента экстинкции $(b)$ тонкой пленки CdSe.

спектра в сторону высоких значений энергий $\left(k_{x, y}<k_{z}\right)$. Такое поведение может быть обусловлено структурными особенностями $(a=b \neq c)$.

\section{Выводы}

Микрофотографии поверхности пленок показали формирование однородных пленок с равномерным распределением зерен соединения. Результаты рентгеноструктурного анализа подтвердили формирование полупроводникового соединения $\mathrm{CdSe}$ (пространственная группа $\left.\mathrm{P6}_{3} m c\right)$.

На спектральных зависимостях оптического пропускания наблюдается наличие края фундаментального поглощения, положение которого соответствует значениям, характерным для соединения $\mathrm{CdSe}$. На спектре оптического поглощения образца $\mathrm{CdSe}$ наблюдается край фундаментального поглощения, на основе которого удалось установить значение оптической ширины запрещенной зоны - $1.68 \mathrm{eV}$.

На основе теоретических расчетов установлено, что тонкая пленка $\mathrm{CdSe}$ обладают прямой запрещенной зоной, локализованной в точке $\mathbf{G}$ зоны Бриллюэна. Обнаружено различие анизотропии между валентной зоной и зоной проводимости $E(\mathbf{k})$. Вершина валентного комплекса более плоская, это вызвано тем, что дырки имеют меньшую подвижность в сранении с электронами. Установлен генезис зоны проводимости и запрещенной зоны.

Рассчитаны основные оптические функции для тонкой пленки CdSe. Наблюдается значительная анизотропия диэлектрических функций в зависимости от оптической поляризации. Анализ коэффициента экстинкции и показателя преломления подтверждает наличие анизотропии $(x=y \neq z)$.

\section{Дополнительная информация}

Расчеты проведены с использованием программного пакета Quantum ESPRESSO [36].

\section{Конфликт интересов}

Авторы заявляют, что у них нет конфликта интересов.

\section{Список литературы}

[1] Zhang Y., Li P., Lau W.M., Gao Y., Zi J., Zheng Z. // Mater. Chem. Phys. 2014. V. 145. P. 441. doi 10.1016/j.matchemphys.2014.02.033

[2] Al-Kotb M.S., Al-Waheidi J.Z., Kotkata M.F. // Superlattices Microstruct. 2014. V. 69. P. 149. doi 10.1016/j.spmi.2014.02.007

[3] Wang X., Li R., Fan D. // Nanoscale Res. Lett. 2011. V. 6. P. 614. doi 10.1186/1556-276X-6-614

[4] Devadason S., Muhamad M.R. // Phys. B Condens. Matter. 2007. V. 393. P. 125. doi 10.1016/j.physb.2006.12.073

[5] Mahawela P., Jeedigunta S., Vakkalanka S., Ferekides C.S., Morel D.L. // Thin Solid Films. 2005. V. 480-481. P. 466. doi 10.1016/j.tsf.2004.11.066

[6] Park Y.M., Andre R., Kasprzak J., Dang L.S., BelletAmalric E. // Appl. Surf. Sci. 2007. V. 253. P. 6946. doi 10.1016/j.apsusc.2007.02.012

[7] Shyju T.S., Anandhi S., Indirajith R., Gopalakrishnan R. // J. Cryst. Growth. 2011. V. 337. P. 38. doi 10.1016/j.jcrysgro.2011.09.051

[8] Larramendi E.M., Karla Gutiérrez Z.-B., O. de Melo, Woggon U., Schikora D., Lischka K. // J. Cryst. Growth. 2010. V. 312. P. 1807. doi 10.1063/1.3374706

[9] Бубнов Ю.З., Лерье М.С., Старос Ф.Г., Филаретов Г.А. Вакуумное нанесение пленок в квазизамкнутом объеме, М.: Советское Радио, 1975.

[10] Бойко Б.Т., Марунько С.В., Панчеха П.А., Полочкий В.А. // Изв. вузов. Физика. 1984. № 1. С. 108-110. 
[11] Калинкин И.П. Эпитаксиальные пленки соединений А2В6. Л.: ЛГУ, 1978.

[12] Il'Chuk G.A., Kurilo I.V., Kus'nezh V.V., Petrus R.Y., Kogut I.T., Stan'ko T.N. // Inorg. Mater. 2013. V. 49. P. 329. doi 10.1134/S0020168513030059

[13] Il'chuk G.A., Kurilo I.V., Petrus R.Y., Kus'nezh V.V. // Inorg. Mater. 2014. V. 50. P. 559. doi 10.1134/S0020168514060077

[14] Ilchuk H.A., Petrus R.Y., Kashuba A.I., Semkiv I.V., Zmiiovska E.O. // Nanosistemi, Nanomater., Nanotehnologii. 2018. V. 16. P. 519

[15] Larramendi E.M., Karla Gutiérrez Z.-B., O. de Melo, Woggon U., Schikora D., Lischka K. // J. Cryst. Growth. 2010. V. 312. P. 1807. doi 10.1063/1.3374706

[16] Ivashchenko M.M., Opanasyuk A.S., Perekrestov V.I., Kosyak V.V., Gnatenko Y.P., Kolomiets V.M. // Vacuum. 2015. V. 119. P. 81. doi 10.1016/j.vacuum.2015.04.036

[17] Baines T. Zoppi G., Bowen L., Shalvey T.P., Mariotti S., Durose K., Major J.D. // Sol. Energy Mater. Sol. Cells. 2018. V. 180. P. 196. doi 10.1016/j.solmat.2018.03.010

[18] Yong-Nian Xu, Ching W.Y. // Phys. Rev. B. 1993. V. 48. P. 4335. doi 10.1103/PhysRevB.48.4335

[19] Hopfield J.J. // J. Appl. Phys. 1961. V. 32. P. 2277. doi 10.1063/1.1777059

[20] Bergstresser T.K., Cohen M.L. // Phys. Rev. 1967. V. 164. P. 1069. doi 10.1103/PhysRev.164.1069

[21] Zakharov O., Rubio A., Blasé X., Cohen M.L., Louie S.G. // Phys. Rev. B. 1994. V. 50. P. 10780. doi 10.1103/PhysRevB.50.10780

[22] STOE \& Cie GmbH, WinXPOW 3.03, Powder Diffraction Software Package, Darmstadt, Germany, 2010.

[23] Brus V.V., Solovan M.N., Maistruk E.V., Kozyarskii I.P., Maryanchuk P.D., Ulyanytsky K.S., Rappich J. // Phys. Solid State. 2014. V. 56. P. 1947. doi 10.1134/S1063783414100072

[24] Khrypunov G.S., Kopach G.I., Dobrozhan A.I., Mygushchenko R.P., Kropachek O.V., Lyubov V.M. // Funct. Mater. 2019. V. 26. P. 23. doi $10.15407 / \mathrm{fm} 26.01 .23$

[25] Ninomiya S., Adachi S. // J. Appl. Phys. 1995. V. 78. P. 4681. doi 10.1063/1.359815

[26] Vanderbilt D. // Phys. Rev. B. 1990. V. 41. P. 7892. doi 10.1103/PhysRevB.41.7892

[27] Perdew J.P., Burke K., Ernzerhof M. // Phys. Rev. Lett. 1996. V. 77. P. 3865. doi 10.1103/PhysRevLett.77.3865

[28] Monkhorst H.J., Pack J.D. // Phys. Rev. B. 1976. V. 13. P. 5188. doi 10.1103/PhysRevB.13.5188

[29] Schlegel H.B. // J. Comp. Chem. 1982. V. 3. P. 214. doi $10.1002 /$ jcc.540030212

[30] Petrus R.Yu., Ilchuk H.A., Sklyarchuk V.M., Kashuba A.I., Semkiv I.V., Zmiiovska E.O. // J. Nano- and Electronic Physics. 2018. V. 10. P. 06042. doi 10.21272/jnep.10(6).06042

[31] Velumani S., Mathew X., Sebastian P.J., Narayandass Sa.K., Mangalaraj D. // Solar Energy Materials and Solar Cells. 2003. V. 76. P. 347. doi 10.1016/S0927-0248(02)00287-8

[32] Kashuba A.I., Piasecki M., Bovgyra O.V., Stadnyk V.Yo., Demchenko P., Fedorchuk A., Franiv A.V., Andriyevsky B. // Acta Physica Polonica A. 2018. V. 133. P. 68. doi 10.12693/APhysPolA.133.68

[33] Kashuba A., Andriyevskyy B., Semkiv I., Andriyevska L., Petrus R., Zmiiovska E., Popovych D. // J. Nano- and Electronic Physics. 2018. V. 10. P. 06025. doi 10.21272/jnep.10(6).06025
[34] Petrus R.Yu., Il'chuk H.A., Kashuba A.I., Semkiv I.V., Zmiiovska E.O. // Opt. and Spectrosc. 2019. V. 126. P. 220. doi 10.1134/S0030400X19030160

[35] Gajdos M., Hummer K., Kresse G., Furthmuller J., Bechstedt F. // Phys. Rev. B. 2006. V. 73. P. 045112. doi 10.1103/PhysRevB.73.045112

[36] Giannozzi P., Baroni S., Bonini N., Calandra M., Car R., Cavazzoni C., Ceresoli D., Chiarotti G.L., Cococcioni M., Dabo I., DalCorso A., Fabris S., Fratesi G., S. de Gironcoli, Gebauer R., Gerstmann U., Gougoussis C., Kokalj A., Lazzeri M., Martin-Samos L., Marzari N., Mauri F., Mazzarello R., Paolini S., Pasquarello A., Paulatto L., Sbraccia C., Scandolo S., Sclauzero G., Seitsonen A.P., Smogunov A., Umari P., Wentzcovitch R.M. // J. Phys.: Condens Matter. 2009. V. 21. P. 395502. doi 10.1088/0953$8984 / 21 / 39 / 395502$ 